\title{
Illness perceptions and work participation: a systematic review
}

\author{
J. L. Hoving $\cdot$ M. van der Meer $\cdot$ A. Y. Volkova $\cdot$ \\ M. H. W. Frings-Dresen
}

Received: 31 July 2009/Accepted: 14 January 2010/Published online: 4 February 2010

(C) The Author(s) 2010. This article is published with open access at Springerlink.com

\begin{abstract}
Purpose Self-regulatory processes play an important role in mediating between the disease and the health outcomes, and potentially also work outcomes. This systematic review aims to explore the relationship between illness perceptions and work participation in patients with somatic diseases and complaints.

Methods The bibliographic databases Medline, PsycINFO and Embase were searched from inception to March 2008. Included were cross-sectional or longitudinal studies, patients with somatic diseases or complaints, illness perceptions based on at least four dimensions of the common sense model of self-regulation, and work participation.

Results Two longitudinal and two cross-sectional studies selected for this review report statistically significant findings for one or more illness perception dimensions in patients with various complaints and illnesses, although some dimensions are significant in one study but not in another. Overall, non-working patients perceived more serious consequences, expected their illness to last a longer time, and reported more symptoms and more emotional responses as a result of their illness. Alternatively, working patients had a stronger belief in the controllability of their condition and a better understanding of their disease.

Conclusions The limited number of studies in this review suggests that illness perceptions play a role in the work participation of patients with somatic diseases or
\end{abstract}

J. L. Hoving $(\bowtie) \cdot$ M. van der Meer · A. Y. Volkova .

M. H. W. Frings-Dresen

Coronel Institute of Occupational Health,

Research Center for Insurance Medicine,

Academic Medical Center, University of Amsterdam,

P.O. Box 22660, 1100 Amsterdam, The Netherlands

e-mail: j.l.hoving@amc.uva.nl complaints, although it is not clear how strong this relationship is and which illness perception dimensions are most useful. Identifying individuals with maladaptive illness perceptions and targeting interventions toward changing these perceptions are promising developments in improving work participation.

Keywords Illness perceptions - Self-regulation return to work - Work participation - Employment . Somatic complaints

\section{Introduction}

For most people, and for most patients with a disease, work remains an important part of life. However, research consistently finds that due to disease, a segment of the patient population does not return to work. The consequences of work disability for patients with chronic diseases include financial difficulties, increased social isolation, decreased confidence and self-esteem and stress. Moreover, it has been shown that once people have been off work for considerable time, they are not likely to return to work. For this reason, more attention is being paid to prevent work disability. There is increasing evidence that psychological factors play an important role in the course of the chronic disease process. In recent years, research concerning chronic diseases suggested that self-regulatory processes play an important role in mediating between disease and health and work outcomes (Leventhal et al. 1997).

When patients are diagnosed with a disease, they develop an organized pattern of beliefs about their health condition (Petrie and Weinman 2006). These beliefs are called illness perceptions and they determine patients' future behavior concerning managing the disease. Disease refers to another 
dimension than illness, namely disease is an objective, medically diagnosed health condition that may lead to disability and incapacity to work. Illness is a subjective feeling of being unwell that is not necessarily accompanied by the diagnosed health condition, but equally may lead to incapacity to work (Waddell et al. 2007).

Illness perceptions have predominantly been described as part of the common sense model of self-regulation and aim to describe the process by which individuals respond to a perceived health threat (Leventhal et al. 1980). The idea behind this model is that individuals are active problem solvers who make sense of a threat to their health by developing their own cognitive representation of the threat, which, in turn, determines how they then respond to it (Petrie and Weinman 2006). The concept of "illness perceptions" has been a focus of many research studies evaluating and predicting patient outcomes in the past decades and has been adapted and advocated by many authors as shown by several reviews (Hagger and Orbell 2003; Coutu et al. 2008; Fadyl and McPherson 2008).

Initially, Leventhal et al. (1980) distinguished five domains considered to be important when assessing these illness representations or perceptions, including (1) the identity of the illness based on the diagnosis or symptoms associated with it; (2) the timeline of the illness (3) the short- and long-term consequences; (4) the factors contributing to the illness and (5) ways to control or cure the illness. Although illness representations were initially assessed using interviews, the drawbacks of this method led to the development of measures such as the Implicit Model of Illness Questionnaire (Turk et al. 1986), the Illness Cognition Questionnaire (Evers et al. 2001) and the Illness Perception Questionnaire (IPQ) (Weinman et al. 1996) or subsequent modifications such as the revised IPQ (IPQ-R) (Moss-Morris et al. 2002) or the brief version of the IPQ (IPQ-B) (Broadbent et al. 2006). These quantitative measures all use the five domains identified by Leventhal, although the revised IPQ (IPQ-R) also further developed the model by including new dimensions, i.e., 'emotional' and 'coherence' representations.

Factors closely linked to several illness representation dimensions have also been used in several other onedimensional or multi-dimensional questionnaires measuring psychosocial dimensions (Coutu et al. 2008). These include questionnaires on catastrophizing (Sullivan et al. 1995), self-efficacy, or attitudes or experiences of pain (Gibson and Strong 1996; Jensen et al. 1987; Edwards et al. 1992), but do not aim to describe all dimensions considered to be important in the link between representations, coping behavior and outcomes as described in the common sense model. Illness perceptions directly influence the individual's emotional response to the disease or complaint and their coping behavior as has been shown in studies on treatment adherence, which could be, for example, a physician's recommendation regarding return to work. The common sense model assumes a causal link between illness representations, the coping strategies patients adopt in response to their illness and the health outcomes of patients. The IPQ and subsequent revisions are based on assessing just the first stage of the common sense model of self-regulation, i.e., interpretation of the cognitive or emotional representation of the health threat.

There is still much unknown about the relationship between illness perceptions and work participation. The knowledge we have suggests illness perceptions could play a role in the employment status of ill people. In this view, 'unhelpfull' or 'maladaptive' illness perceptions would result in reduced work participation (i.e., more sickness absenteeism, work disability and unemployment) and economic or social deprivation. In the absence of (regular) work, a person lacks not only a place in which to work and the receipt of regular income but also a coherent structure of everyday life and goals. In contrast, positive or 'helpful' illness perceptions could play an important role in returning to work with an illness. Research shows that illness perceptions affect functional adaptation and adherence to medical rehabilitation (Heijmans 1998; Orbell et al. 1998; Scharloo et al. 1998). Therefore, evaluating and bolstering the patients' beliefs about their health conditions may be an important component of the vocational rehabilitation process.

As far as we can ascertain there are no systematic reviews evaluating the relationship between illness perceptions and work participation in patients with somatic complaints or diseases. Therefore, this paper explored the relationship between illness perceptions and work participation in patients with somatic diseases and complaints by reviewing the literature.

Where possible, we will discuss and expand on the role of illness perceptions within the occupational health setting. Better understanding of the role of illness perceptions in the occupational health setting would aid its potential use in the design and analysis in clinical trials (e.g., risk stratification), for adjustment (of particular importance in observational studies), in defining high risk groups (based on prognosis), or assist in decision-making during the selection of appropriate interventions or patient counseling.

\section{Materials and methods}

Search strategy

The search strategy comprised a search of computerized bibliographic databases (PubMed, PsycINFO and Embase) from inception to March 2008 using both subject headings such as MeSH terms (PubMed) and free text words. 
The terms selected to identify studies were grouped in two main categories, i.e., terms identifying the factor of interest i.e., illness perceptions, and terms to identify terms on work participation (outcomes), and then combined with the Boolean operator 'AND'. Combinations of terms on illness perceptions included: illness perceptions, illness representations, cognitive representations, illness cognitions, self-regulation. Search terms on work-related outcomes included employment, work, participation, work disability, return to work, occupational, absenteeism and have been described by Haafkens et al. (2005). When available, the references of the included articles and recently published review articles were screened for additional publications. An example of the full search strategy in Pubmed is provided in Appendix 1.

Search strategy and study selection

Studies were included in the review if:

1. a cross-sectional or longitudinal design was used;

2. the study population concerned patients with somatic diseases or complaints at inclusion;

3. illness perceptions were measured using a questionnaire that contained at least four dimensions of the CSM-model of self-regulation such as identity of the illness, beliefs about cause of the illness and about how long it will last, beliefs about personal consequences of the condition, and/or beliefs about personal control; and,

4. the study used work participation as an outcome of interest, including employment status (employed versus not employed, sick listed or work disabled), return to work or days absent from work.

In the first round, two investigators independently reviewed all titles and abstracts of the identified publications and excluded all studies that did not fulfill one or more selection criteria. If the abstract was non-informative but potentially relevant, the full text article was read. In the second round, full text articles were ordered and studies were selected if they fulfilled all four criteria. Selection was performed independently by two reviewers.

\section{Data extraction and study quality}

Data extraction was performed by one reviewer and checked by another and was performed using a checklist that included items on social demographic characteristics of the study population (age, gender, diagnosis or somatic diseases or complaints and employment status), sample size, outcome measures concerning work participation, duration of follow-up and results of the most important illness perception categories reported in the studies obtained from the descriptive analyses or regression analyses.

Study quality was independently assessed by two reviewers using a methodology checklist from NICE (National Institute for Health and Clinical Excellence) adapted from Hayden et al. (2006) to assess whether key study information was reported and the risk of bias was minimized (scoring yes, no or unclear), based on the following topics: (a) study sample representativeness (description key characteristics, source population, sampling and recruitment methods), (b) loss to follow up/ response rate (description of: rate of drop outs and reasons, loss to follow up and reasons, differences in key characteristics), (c) measurement of illness perceptions/dimensions (valid and well defined, used well-developed measurement tool to measure factor of interest), (d) measurement of work participation (well defined, methods for assessing outcome are valid and reliable) (e) accounting for potential confounders (confounders are described, measured and accounted for in analyses). The quality scores will be presented and discussed separately. A full description of all items is available from the authors. Only items fulfilling a criterion received a plus ("yes") score.

\section{Analyses}

Provided the studies were sufficiently homogeneous, and data analyses and data presentation were complete, we planned a meta-analyses. Otherwise, data were discussed qualitatively, considering all key characteristics and placing the evidence in light of the study strengths and weaknesses. To best explain the relationship between illness perceptions and work participation, we made a distinction between studies with a longitudinal design and those with a cross-sectional design. As the design of longitudinal studies carries, in comparison with cross sectional studies, in potential more weight with regard to causality, these are presented first.

The results were described by considering both the type of analyses (descriptive analyses or multivariate analyses) and the type of study design (longitudinal or cross-sectional design). Both the longitudinal studies and the crosssectional studies used descriptive (comparative) analyses by comparing illness perception dimension scores in working versus non-working patients. In addition, both also used multivariate stepwise regression analyses to show the added value of including illness perceptions over and above commonly used health and socio-demographic variables, either in predicting return to work using baseline data (longitudinal studies) or in showing its association with work participation (cross-sectional studies) at one moment in time. 


\section{Results}

Study selection and characteristics

The primary search strategy generated 5,163 references. After a first selection on title and abstract, 158 references were left for full-text screening. The majority of studies were excluded as they did not include an outcome on the level of work participation. Four studies met all criteria for inclusion and were selected for this review; two small studies using a longitudinal design including 72 and 77 patients (Petrie et al. 1996; McCarthy et al. 2003) and two larger survey studies using a cross-sectional design including 552 and 1,121 subjects (Sluiter and Frings-Dresen 2008; Boot et al. 2008). The study populations in the two longitudinal studies by McCarthy et al. (2003) and Petrie et al. (1996) included, respectively, recent trauma as a result of molar extractions in the past week or recent myocardial infarction in the past 6 weeks. The two crosssectional survey studies by Boot et al. (2008) and Sluiter and Frings-Dresen (2008) both included chronic populations: one with various chronic diseases (mean duration $8-$ 10 years) (Boot et al. 2008) and the other chronic repetitive strain injury (RSI) (mean pain duration 6 years) (Sluiter and Frings-Dresen 2008) (see Table 1). The outcomes of work participation and definitions differed between studies; i.e., days until back to work, return to work rates at 6 weeks (longitudinal studies), or sick-listed or fully work disabled (cross-sectional studies). All four studies used the illness perceptions questionnaire or modifications thereof (IPQ, IPQ brief, IPQ revised) (see Table 1), and each study reported a different number of questionnaire items and scale characteristics (different number of questions and different Likert scale ranges). As can be seen from Table 1, studies did not meet all quality criteria, with the exception of the Boot et al. (2008) study. Both in Petrie et al. (1996) and Sluiter and Frings-Dresen (2008), information on the source and study population was missing, including (reasons for) loss to follow up (27\% in Petrie et al. 1996) and a low response rate $(36 \%$ response rate in Sluiter and Frings-Dresen 2008) resulted in not fulfilling these criteria. In addition, in two studies, potential confounders such as age, disease duration, or disease severity were not presented or accounted for in the analyses (Petrie et al. 1996; Sluiter and Frings-Dresen 2008).

\section{Data analyses and outcomes}

Regardless of the analyses methods used, all studies report statistically significant findings for one or more illness perception dimensions (Table 1). A few studies did not use all illness dimensions of the IPQ or subsequent versions in the analyses hence some dimensions are more frequently reported, including the 'consequences' dimension, 'timeline' dimension, and the 'control' dimension. Although the direction of the effects for the individual illness perception dimensions was generally in the same direction, some were significant in one study but not in the other study. As data analyses, data presentation and study quality varied considerably, direct comparisons between studies presenting absolute point estimates and studies presenting regression parameters are less informative. Considering the heterogeneity between studies, we considered pooling of the results not feasible and evaluated the results of the studies qualitatively.

In the three studies reporting descriptive analyses, overall higher scores on the dimension consequences, timeline, identity and concern were observed in the nonworking groups reflecting a negative relationship, whereas higher scores on the dimensions' control and coherence reflected a positive relationship on work participation as seen in the working group (Petrie et al. 1996; Boot et al. 2008; Sluiter and Frings-Dresen 2008). The result of the causal dimension was not reported in most studies, except for the study by Boot et al. (2008) because this scale often consisted of open questions. Although all illness dimensions showed differences of various magnitudes indicating more maladaptive beliefs in the non-working group, some were not statistically significant. The magnitude of the differences between groups were small; for example, those who did not work rated the consequences of their disease on average between 1 and 2 points more severe (on 0-10 scale) (Boot et al. 2008; Sluiter and Frings-Dresen 2008) compared to those who did work. The magnitudes of the differences for the other illness perception dimensions, albeit in the same direction, were smaller.

In the study by Petrie et al. (1996) on recently admitted patients with a first myocardial infarction, the absolute scores in two out of four illness perception dimensions, i.e., timeline and consequences, showed statistically significant differences between the group who returned to work within six weeks and a group who took longer than six weeks. The study by Boot et al. (2008) on patients with chronic diseases also showed that all five included dimensions from the revised IPQ showed maladaptive illness representations were more severe in the group that was fully work disabled versus the group that was employed. Sluiter and FringsDresen (2008) also compared differences in several illness perceptions measured on the IPQ-brief in sick-listed patients versus working patients with repetitive strain injury (RSI). Except for the dimensions 'timeline', all differences between groups were statistically significant. The authors also reported that the dimensions 'consequences', 'personal' and 'treatment control', and 'identity' were "clinically important" in terms of effect size, i.e., a difference of 1 point on a 10 point scale. 


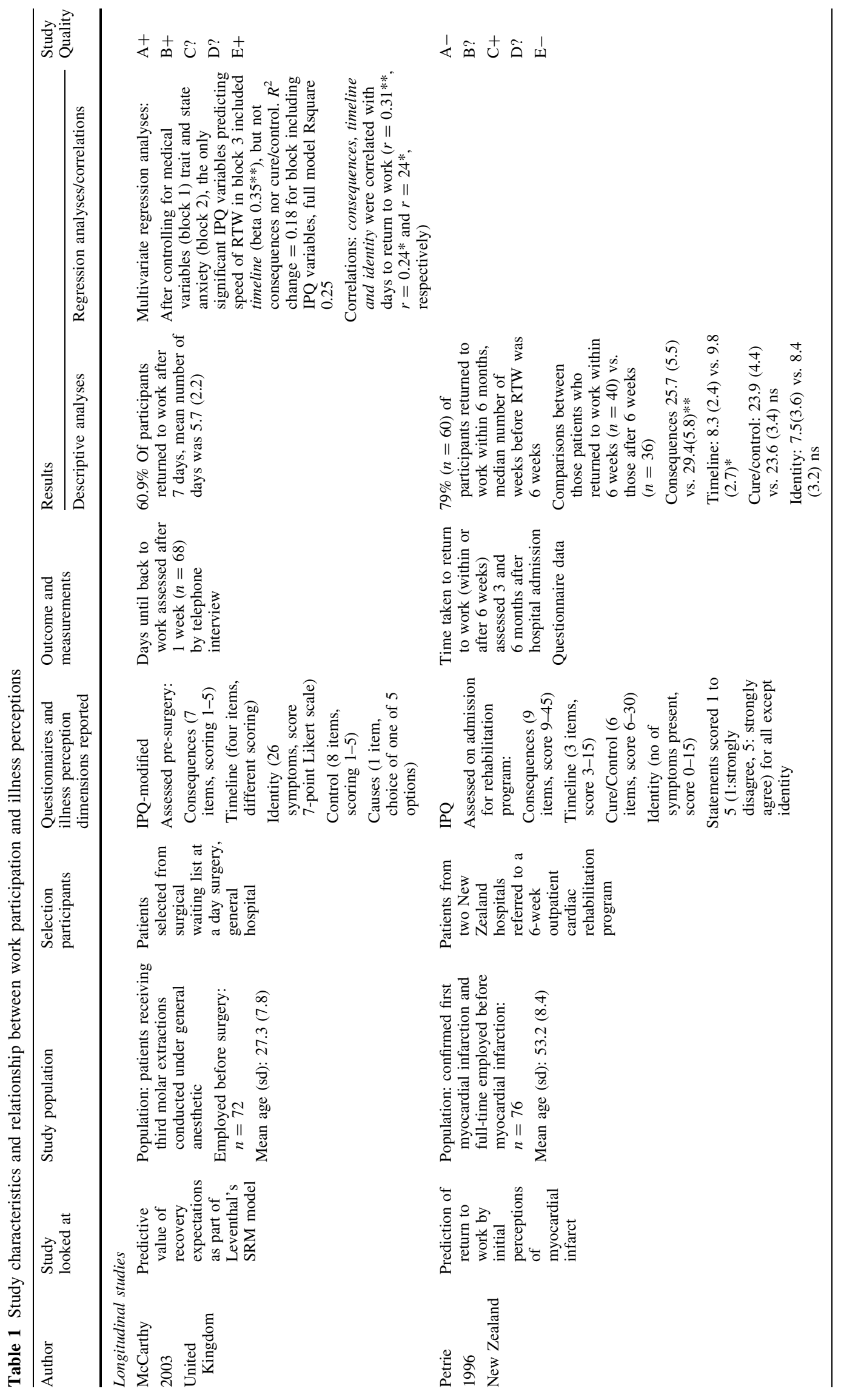




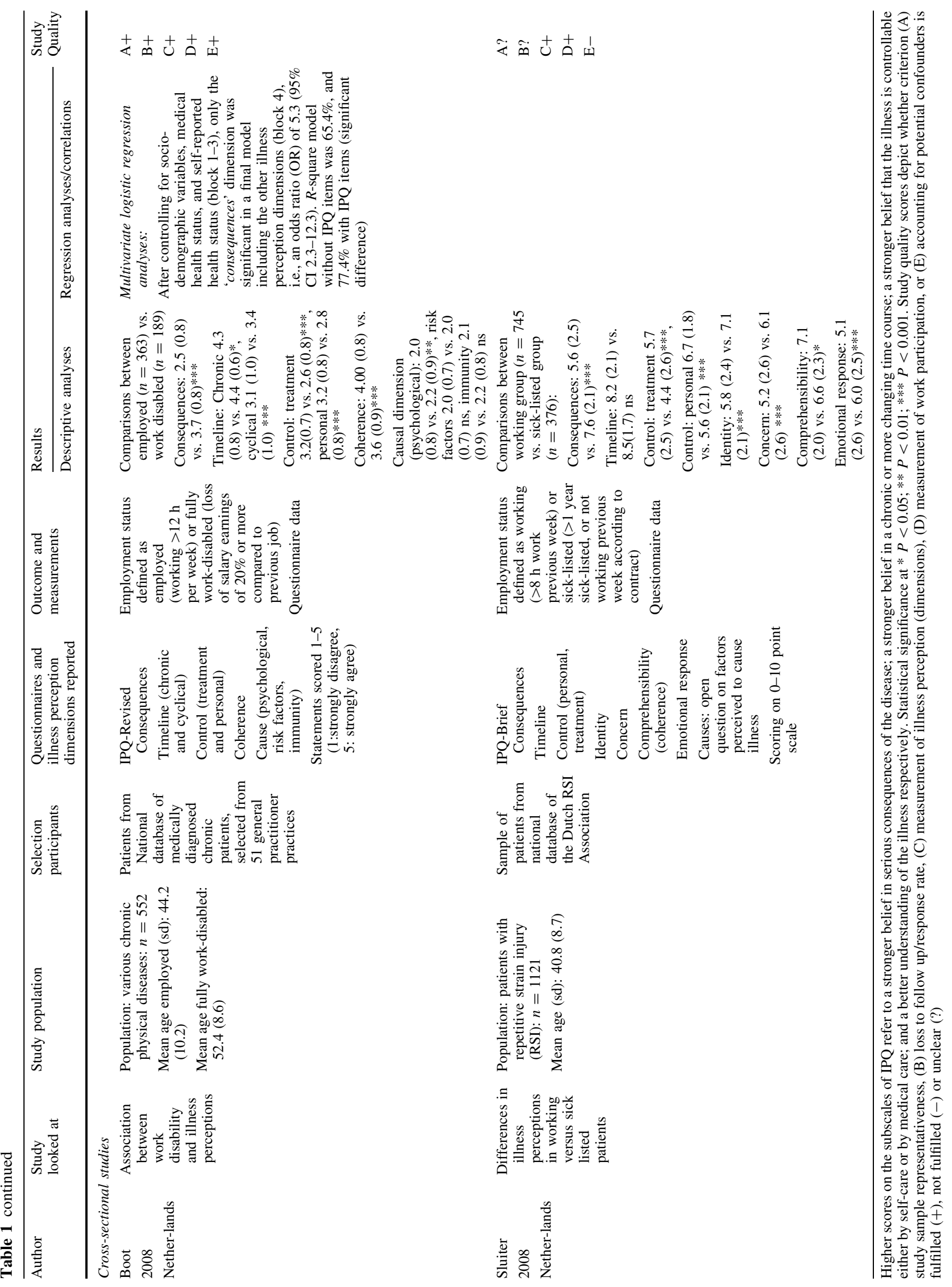


In the two cross-sectional and longitudinal studies reporting regression analyses (Boot et al. 2008; McCarthy et al. 2003), no univariate associations are presented, hence individual associations between the different illness perception dimensions and work participation cannot be assessed. Although several illness perception dimensions were included after the inclusion of socio-demographic, medical and health outcome variables, two dimensions emerged from the final multivariate models. McCarthy et al. (2003) showed that the pre-operative question on the dimension timeline, i.e., "how many days it would take for normal functioning to return", was the only illness perception item to predict the number of days taken to return to work in a multivariate stepwise regression model adjusted for medical and anxiety factors (beta 0.35 , $P<0.01)$. Similarly, the multivariate logistic regression analyses by Boot et al. (2008) showed that the dimension consequences within the last model including all illness perception dimensions, had a strong association with employment status as reflected by a large odds ratio of 5.3 (95\% CI 2.3-12.3). The inclusion of the dimension timeline in the study by McCarthy et al. (2003) or the dimension consequences in combination with the other illness perceptions in the study by Boot et al. (2008), showed an increase in the explained variance of, respectively, $18 \%$ (beta 0.35) (from 7 to 25\%) (McCarthy et al. 2003) and almost $10 \%$ (from 65.4 to $77.4 \%$ ) (Boot et al. 2008).

\section{Conclusion and discussion}

In this systematic review, we explored the relationship between illness perceptions and work participation. We found promising evidence in four studies to suggest that illness perceptions play a role in the work participation of patients with somatic diseases or complaints, even though extrapolation is limited given the low number of comparable studies and analyses. Notwithstanding heterogeneity, methodological quality issues and the limited evidence presented, all studies report significant findings between one or more illness perception dimensions and measures of work participation.

Descriptive analyses show non-working people perceive more negative consequences of their illness, which was reported in both cross-sectional and longitudinal studies. The other illness perception dimensions were significant in some but not in all studies. In the hierarchical multivariate analyses, the added benefit of the illness perception dimensions consequences (McCarthy et al. 2003) or timeline (Boot et al. 2008), above that for other socio-demographic and medical variables was shown, of which only McCarthy et al. (2003) showed a temporal relationship. From the latter longitudinal study (McCarthy et al. 2003), it can be deducted, even for a relatively short period of sick leave and independent of other factors, how the score on the timeline scale is related to real sick leave. One-day increase in patients' expectations of return to normal activities will also increase sick leave by $1 / 3$ day, independent of other factors. Based on the results in above, it would be interesting to further investigate which individual illness perception dimensions or which combinations of illness perception dimensions would best predict future work disability in patients and target these with interventions at an early stage, if possible.

Our review shows that illness perceptions play a role across several illnesses, ranging from acute trauma to chronic diseases. One could ask whether the relationship between illness perception and work participation depends on the type of complaints or disease. Although illness perception dimensions play a role in many diseases, the degree to which patients have 'unhelpful' or 'maladaptive' illness perceptions varies. For example, differences in the severity of maladaptive illness perception dimensions have been found between patients with fibromyalgia, chronic fatigue syndrome, rheumatoid arthritis and coronary heart disease (Moss-Morris and Chalder 2003; van Ittersum et al. 2009). However, whether this also affects the strength of the relationship between illness perceptions and work participation remains to be seen and is not evident from this review. Similarly, it has been suggested that later in the course of the disease, as opposed to more acute conditions, symptoms and disability levels stabilize as recovery is slowing down, which may provide weaker associations between illness perceptions and work participation (compared to acute disease) (Iles et al. 2009) but we did not observe this difference in our small sample of studies.

A few comments can be made about the instruments used to measure illness perceptions in this review before their application or practical use is considered. Firstly, the revised IPQ (Moss-Morris et al. 2002; Broadbent et al. 2006) or a shorter version, the IPQ brief, may be preferred due to their improved psychometric properties over that of the original illness perceptions questionnaire (Weinman et al. 1996). Secondly, the illness perception questionnaire most often needs further modification to be useful for a particular disease or cultural setting, in particular for the causal and identity scales (Moss-Morris and Chalder 2003). This is illustrated in the study by McCarthy et al. (2003) who changed the IPQ scale characteristics considerably, although it is not clear whether this also influenced the strength of the associations in any direction. This highlights the need for psychometric testing of the IPQ and subsequent versions for different diseases and settings, in particular if substantial revisions are made (French and Weinman 2008). Thirdly, it is suggested that the illness perception dimensions are not used in isolation (Leventhal 
and Cameron 1987), but interpreted as a whole or in subsets or profiles to be useful in practice (French and Weinman 2008), which may be different from its use in prediction studies where typically only the strongest predictors (i.e., single dimensions) are of interest. Both for clinical practice and for research purposes, the use and interpretation of absolute illness perception scores could be improved, however, especially if cut-off values were to be proposed and normative data would help to distinguish 'helpful' from 'unhelpful' illness perceptions in different diseases and settings. In addition, it will be of interest to investigate whether combinations of illness perception dimensions show stronger relationships with work disability when compared to single dimensions.

Illness perceptions and patient expectation beliefs show promise in predicting health and work participation outcomes in several other studies. In a meta-analysis of 45 studies, Hagger and Orbell (2003) showed that there are predictable relations between illness representations, illness coping behavior and outcomes across studies and across different illness types. A link between illness representations and health outcomes was shown for the dimensions 'consequences', 'identity' and 'timeline' which all showed a negative relationship with quality of life dimensions such as psychological well-being, role and social functioning, and vitality (Hagger and Orbell 2003). These three dimensions were frequently applied in our review and showed significant differences in the descriptive analyses although not consistently across all studies, except for the consequences dimension. This review adds to the growing body of evidence in showing that ideas and expectations patients have about their illness and recovery are good predictors of future health outcomes and functioning. Patients who have poor recovery expectations report more disability, return to work less fast and have more pain over time compared to patients with good recovery expectations (Iles et al. 2008, 2009). Similarly, other related psychological factors such as catastrophizing beliefs, thought to be a component of the illness perception dimensions identity, controllability and consequences (Hobro et al. 2004), may also influence return to work outcomes (Fadyl and McPherson 2008). Therefore, bolstering patient's beliefs about their present or future health condition and their ability to work seems important.

Although counseling and cognitive behavioral therapy have been used in many return to work programs to improve patients' coping strategies, its explicit use in focusing on 'dysfunctional' illness representations, or socalled 'self-regulatory illness management' (McAndrew et al. 2008), has gained interest in intervention studies, including randomized trials. Interventions based on the common sense model of self-regulation have the advantage of being theory driven, individualized, patient-centered and have been suggested to involve both cognitive and behavioral components (Wearden and Peters 2008). This model shows how poor self-regulation is maintained in persons with an illness but also shows that cognitive and behavioral skills can be adopted to change behavior and confront maladaptive cognitions. Several intervention studies have adopted the concept of the common sense model of self-regulation in both the design of the interventions and its use as a measure of effect in assessing illness representations.

There are some good examples showing that illness perceptions, as described in common sense model, can be used to target intervention strategies in patients with various diseases, and with good results. A good example of an intervention due to its focus on work participation is provided by the randomized controlled trial of Petrie et al. (2002), who showed significantly faster return to work rates in an experimental group of post-myocardial infarction patients receiving counseling by a psychologist that focused on changing illness perceptions compared to a control group that did not. Patients modified their perceptions about how long their illness would last and reappraised the personal consequences of the myocardial infarction on their life. The strength of the program was that the individual scores of the illness perception questionnaire were used as a starting point for the intervention, that it was theory based, individualized, structured and not fixed on a number of standard 'one fits all' rehabilitation intervention components. Several other intervention studies specifically addressing illness representations also showed that illness representations can be positively targeted by various professionals, in different mode intensities or frequencies, and for patients with various diseases like lupus erythematosus (Goodman et al. 2005), psoriasis (Fortune et al. 2004) or essential hypertension (Theunissen et al. 2003). As illustrated by the emerging evidence in these intervention studies, interaction between a patient and a general practitioner, psychologist or nurse by stimulating interaction on expectations and beliefs about the disease can reduce unhelpful perceptions, improve coping skills and improve health or work outcomes.

In the occupational health setting, more attention for illness perceptions by health professionals seems therefore sensible. Many health professionals are unaware of the relevance of discussing patient's illness representations or strategies patients adopt to deal with their illness. At the same time, patients do not often spontaneously articulate these issues if they are not encouraged to do so. Discussing illness perceptions is appreciated by patients and create a feeling of support (Theunissen et al. 2003). Preventative actions can be taken by an occupational professional for a worker who is at risk of dropping out with an illness. This could include offering more positive views about the 
illness and possibilities to work, provide ability to vent emotions, encourage social support and communication with the supervisor, and train problem-focused coping at work. Identifying which patients develop maladaptive illness representations would be helpful for health professionals.

It seems sensible to target interventions by (occupational) health professionals to (patterns of) maladaptive illness representations. For example, if patients have unhelpful perceptions regarding the consequences of their illness than the aim could be to help the patient understand these and filter out any unrealistic scenario's. The same applies when patients have unrealistic perceptions of the chronic or recurrent timeline of their illness, or work participation is unnecessarily postponed as only the negative consequences of work are considered by the patient. Also, providing information on occupational interventions or job accommodations could empower a patient to keep working with a chronic disease and boost the patient's perception to control the negative effects of the illness while at work. The above would require the health professional to have an adequate knowledge of the effects that different illnesses have on functioning or more specific work participation, and more importantly, how any of these cognitive or emotional representations can be accommodated for or trained by the worker. This would require skills in cognitive and behavioral therapy, which may be feasible as shown in the Theunissen et al. (2003) who provided GP trainees with a short $(6 \mathrm{~h})$ training in these principles. Other promising vocational rehabilitation strategies are increasingly used in the occupational health field (Hoving et al. 2009; Verbeek 2006) and would benefit from including the concept of illness perceptions. The use of illness perception measures by health professionals would also target specific interventions to those who need it, in contrast to offering the same treatment to everyone, and would be a potential cost-effective option.

It is recommended that any studies investigating the longitudinal relationship between illness perceptions and work participation would use a longitudinal design and appropriate analyses. The use of the common sense model of self-regulation is gaining interest, and the use of illness representations in the occupational health setting should be further explored. Given the limited number, the variable quality of studies and the reported heterogeneity, we would be careful at this point to exclude any 'non-significant' illness perception dimensions from further study in the relationship between work participation and illness perception. However, identification of individuals with abnormal illness perceptions in occupational health is only a first step, it would be sensible to assess whether these illness perceptions can be changed at all, by whom and at what cost.
Conflict of interest statement The authors declare that they have no conflict of interest.

Open Access This article is distributed under the terms of the Creative Commons Attribution Noncommercial License which permits any noncommercial use, distribution, and reproduction in any medium, provided the original author(s) and source are credited.

\section{Appendix 1}

Search strategy in medline (pubmed)

("Work"[Mesh] OR “Employment"[Mesh]OR “Job Satisfaction"[Mesh] OR "Absenteeism"[Mesh] OR "Rehabilitation, Vocational"[Mesh] OR “Sick Leave"[Mesh] OR "Vocational Guidance"[Mesh] OR "Occupational Health Services"[Mesh] OR “Convalescence”[Mesh] OR "work functioning"[All Fields] OR "work adjustment"[All Fields] OR "work rehabilitation"[All Fields] OR "return to work"[All Fields] OR "re-employment"[All Fields] OR “job outcomes”[All Fields] OR "work outcome"[All Fields] OR "work outcomes"[All Fields] OR "work capacity"[All Fields] OR “work participation"[All Fields] OR “work recovery"[All Fields] OR "work status"[All Fields] OR "sick leave"[All Fields] OR "sickness absence"[All Fields] OR "employment status"[All Fields] OR “work disability"[All Fields] OR “work ability"[All Fields] OR “job status"[All Fields] OR "work situation"[All Fields] OR "employment"[MeSH Terms] OR employment[Text Word] OR "unemployment"[MeSH Terms] OR unemployment[Text Word] OR "absenteeism" [MeSH Terms] OR absenteeism[Text Word] OR "vocational rehabilitation"[All Fields] OR "vocational outcome"[All Fields] OR "vocational guidance"[All Fields] OR “job performance"[All Fields] OR "disability pension"[All Fields] OR "economic status"[All Fields] OR "economic consequences"[All Fields] OR "workrelated"[All Fields] OR "work factors"[All Fields] OR "labour participation"[All Fields] OR "labor participation"[All Fields] OR "occupational outcome"[All Fields] OR “occupational status"[All Fields] OR “occupational rehabilitation"[All Fields] OR "occupational adjustment"[All Fields]) AND ("illness representations"[All Fields] OR "illness representation"[All Fields] OR “illness perceptions"[All Fields] OR “illness perception"[All Fields] OR "illness attitude"[All Fields] OR "illness attitudes"[All Fields] OR "illness schema"[All Fields] OR "illness cognition"[All Fields] OR "illness cognitions"[All Fields] OR "illness beliefs"[All Fields] OR "illness model"[All Fields] OR "illness models"[All Fields] OR "perceived illness"[All Fields] OR "causal attribution"[All Fields] OR “causal attribution"[All 
Fields] OR "self-perceived health"[All Fields] OR "IPQR"[All Fields] OR "leventhal"[All Fields] OR "patient expectation"[All Fields] OR "patient expectations"[All Fields] OR "cognitive representation"[All Fields] OR "cognitive representations"[All Fields] OR "attitude to health"[All Fields] OR "recovery expectations"[All Fields] OR "illness perception questionnaire"[All Fields] OR "health appraisal"[All Fields] OR "disease perception"[All Fields] OR "disease perceptions"[All Fields] OR "self-regulatory model" [All Fields] OR self-regulation[Text Word] OR "health attitudes"[All Fields] OR "illness behavior"[All Fields] OR "illness representations questionnaire"[All Fields] OR IMIQ[All Fields] OR "disability perception"[All Fields]).

\section{References}

Boot CR, Heijmans M, van der Gulden JW, Rijken M (2008) The role of illness perceptions in labor participation of the chronically ill. Int Arch Occup Environ Health 82:13-20

Broadbent E, Petrie KJ, Main J, Weinman J (2006) The brief illness perception questionnaire (B-IPQ). J Psychosom Res 60:631-637

Coutu MF, Durand MJ, Baril R, Labrecque ME, Ngomo S, Côté D, Rouleau A (2008) A review of assessment tools of illness representations: are these adapted for a work disability prevention context? J Occup Rehabil 18:347-361

Edwards LC, Pearce SA, Turner-Stokes L, Jones A (1992) The pain beliefs questionnaire: an investigation of beliefs in the causes and consequences of pain. Pain 51:267-272

Evers AWK, Van Lankveld W, Jongen P, Jacobs J, Bijilma J (2001) Beyond unfavorable thinking: the illness cognition questionnaire for Chronic diseases. J Consult Clin Psychol 69:1026-1036

Fadyl J, McPherson K (2008) Return to work after injury: a review of evidence regarding expectations and injury perceptions, and their influence on outcome. J Occup Rehabil 18:362-374

Fortune DG, Richards HL, Griffiths CE, Main CJ (2004) Targeting cognitive-behaviour therapy to patients' implicit model of psoriasis: results from a patient preference controlled trial. Br J Clin Psychol 43:65-82

French DP, Weinman J (2008) Editorial. Assessing illness perceptions: beyond the IPQ. Psych Health 23:5-9

Gibson L, Strong J (1996) The reliability and validity of a measure of perceived functional capacity for work in chronic back pain. $\mathrm{J}$ Occup Rehabil 6:159-175

Goodman D, Morrissey S, Graham D, Bossingham D (2005) Illness representations of systemic lupus erythematosus. Qual Health Res 15:606-619

Haafkens J, Moerman C, Schuring M, van Dijk F (2005) Searching bibliographic databases for literature on chronic disease and work participation. Occup Med (Lond) 56:39-45

Hagger MS, Orbell S (2003) A meta-analytic review of the commonsense model of illness representations. Psychol Health 18:141184

Hayden JA, Côté P, Bombardier C (2006) Evaluation of the quality of prognosis studies in systematic reviews. Ann Intern Med 144:427-437

Heijmans MJ (1998) Coping and adaptive outcome in chronic fatigue syndrome: importance of illness cognitions. J Psychosom Res 45:39-51
Hobro N, Weinman J, Hankins M (2004) Using the self-regulatory model to cluster chronic pain patients: the first step towards identifying relevant treatments? Pain 108:276-283

Hoving JL, Broekhuizen ML, Frings-Dresen MH (2009) Return to work of breast cancer survivors: a systematic review of intervention studies. BMC Cancer 9:117

Iles RA, Davidson M, Taylor NF (2008) Psychosocial predictors of failure to return to work in non-chronic non-specific low back pain: a systematic review. Occup Environ Med 65:507-517

Iles RA, Davidson M, Taylor NF, O'Halloran P (2009) Systematic review of the ability of recovery expectations to predict outcomes in non-chronic non-specific low back pain. J Occup Rehabil 19:25-40

Jensen MP, Karoly P, Huger R (1987) The development and preliminary validation of an instrument to assess patients' attitudes toward pain. J Psychosom Res 31:393-400

Leventhal H, Cameron L (1987) Behavioral theories and the problem of compliance. Patient Educ Couns 10:117-138

Leventhal H, Meyer D, Nerenz D (1980) The commonsense representation of illness danger. In: Rachman S (ed) Contributions to medical psychology, vol 2. Pergamon, Oxford, pp 7-30

Leventhal H, Benyamini Y, Brownlee S, Diefenbach M, Leventhal EA, Patrick-Miller L, Robitaille C (1997) Illness representations: theoretical foundations. In: Petrie KJ, Weinman J (eds) Perceptions of health and illness. Harwood Academic Press, Amsterdam, pp 19-46

McAndrew LM, Musumeci-Szabó TJ, Mora PA, Vileikyte L, Burns E, Halm EA, Leventhal EA, Leventhal H (2008) Using the common sense model to design interventions for the prevention and management of chronic illness threats: from description to process. Br J Health Psychol 13:195-204

McCarthy SC, Lyons AC, Weinman J, Talbot R, Purnell D (2003) Do expectations influence recovery from oral surgery. An illness representation study. Psychol Health 18:109-126

Moss-Morris R, Chalder TJ (2003) Illness perceptions and levels of disability in patients with chronic fatigue syndrome and rheumatoid arthritis. Psychosom Res 55:305-308

Moss-Morris R, Weinman J, Petrie KJ, Horne R, Cameron LD, Buick D (2002) The revised illness perception questionnaire (IPQ-R). Psychol Health 17:1-16

Orbell S, Johnston M, Rowley D, Espley A, Davey P (1998) Cognitive representations of illness and functional and affective adjustment following surgery for osteoarthritis. Soc Sci Med 47:93-102

Petrie KJ, Weinman J (2006) Why illness perceptions matter. Clin Med 6:536-539

Petrie KJ, Weinman J, Sharpe N, Buckley J (1996) Role of patients' view of their illness in predicting return to work and functioning after myocardial infarction: longitudinal study. BMJ 312:11911194

Petrie KJ, Cameron LD, Ellis CJ, Buick D, Weinman J (2002) Changing illness perceptions after myocard infarction: an early intervention randomized controlled trial. Psychosom Med 64:580-586

Scharloo M, Kaptein AA, Weinman J, Hazes JM, Willems LN, Bergman W, Rooijmans HG (1998) Illness perceptions, coping and functioning in patients with rheumatoid arthritis, chronic obstructive pulmonary disease and psoriasis. J Psychosom Res 44:573-585

Sluiter JK, Frings-Dresen MH (2008) Quality of life and illness perception in working and sick-listed chronic RSI patients. Int Arch Occup Environ Health 81:495-501

Sullivan MJR, Bishop SR, Pivik J (1995) The pain catastrophizing scale development and validation. Psych Assessment 7:524-532

Theunissen NC, de Ridder DT, Bensing JM, Rutten GE (2003) Manipulation of patient-provider interaction: discussing illness 
representations or action plans concerning adherence. Patient Educ Couns 51:247-258

Turk DC, Rudy TE, Salovey P (1986) Implicit models of illness. J Behav Med 9:453-474

van Ittersum MW, van Wilgen CP, Hilberdink WK, Groothoff JW, van der Schans CP (2009) Illness perceptions in patients with fibromyalgia. Patient Educ Couns 74:53-60

Verbeek JH (2006) How can doctors help their patients to return to work? PLoS Med 3(3):e88
Waddell G, Burton K, Aylward M (2007) Work and common health problems. J Insur Med 9:109-120

Wearden A, Peters S (2008) Editorial: therapeutic techniques for interventions based on Leventhal's common sense model. Br J Health Psychol 13:189-193

Weinman J, Petrie KJ, Moss-Morris R, Horne R (1996) The illness perception questionnaire: a new method for assessing the cognitive representation of illness. Psychol Health 11:431-435 